\title{
Analysing Voluntary Contribution To Online Forums Using A Proposed Critical Mass Contribution Model
}

Qunying Huo, ESC Rennes School of Business, France

Adrian Palmer, ESC Rennes School of Business, France

\begin{abstract}
Online forums have become a popular means of communication, embracing the concept of "customer co-creation" defined by service dominant logic. Many company sponsored online forums fail to grow, whereas others rapidly achieve a scale at which they become sustainable. There have been many narrative descriptions to propose reasons for success and many partial models based on network growth and random association. This paper makes a contribution to knowledge by proposing a model based on critical mass theories. This integrates a range of discipline bases and recognises the differential rates of contribution of contributors and the evaluations they make prior to contribution. Practical suggestions are made for how companies can use the principles of critical mass models to improve their "seeding" of new forums with a view to rapidly reaching a critical mass where a forum becomes sustainable.
\end{abstract}

Keywords: "Online Forums"; "Critical Mass"; "Customer Co-Creation"

\section{INTRODUCTION}

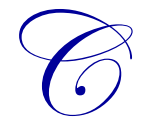

ommunication channels between companies and their customers have become transformed from predominantly bilateral to increasingly multi-lateral, in which technology allows customers to talk to their peers through various social network media. Online forums have emerged which can provide mutual support between customers, for example in resolving queries which might otherwise have been resolved bilaterally between a company and an individual customer. This "co-creation of value" (Vargo and Lusch, 2008a) presumes a willingness and ability of fellow consumers to sacrifice time and effort to produce benefits which may be appropriated by others, and not necessarily reciprocated (Wasco, Teigland and Faraj, 2009).

Online forums play an increasingly important role in business and society (Füller et al., 2008; Dholakia et al., 2009; Demange, 2010). One challenge faced by an electronic forum is the availability of knowledge/ information (Harris and Rae, 2009; He, Qiao and Wei, 2009; Levy, 2009; Payne et al., 2009). Without continuing contributions, visitors would be less likely to visit a forum, and with fewer visitors, contributors of information may be less motivated to post comments, believing that their comments will not have significant impact if the audience is small. However, little research has been undertaken to understand at which level there are sufficient numbers of members producing knowledge which can further ensure others' contribution. And following from this, why do some forums survive into a sustainable state, while others fail to grow and eventually go into decline or disappear?

This study seeks to make a contribution to knowledge by proposing a model of critical mass contribution to answer two main questions.

1) Why do members voluntarily contribute knowledge to online forums?

2) How can the structure of online networks be managed so that critical components (or "hubs" / "giant component") can be present and further ensure sustainable contribution in Web-based networks? 
Against this background, this paper proposes an integrative model which is embedded in a theory of voluntary contribution and considers the dynamic structure of networks to fill the knowledge gaps identified above. The contributions of this study are therefore, to firstly investigate antecedents of voluntary mass contribution online, and secondly to provide a different method to understand the impact of the structure of networks on members' contribution behaviours. This research reflects a pioneering stream of research in online network analysis which takes into account human factors as well as complex network structures.

This paper is structured as follows: The literature review firstly discusses problem recognition and voluntary contribution behaviours in the context of online forums, and a critical mass contribution model is proposed. Following this are descriptions of network analysis highlighting the conditions for phase transition and identification of a giant component which can ensure the stable and continuous expansion of a complex network. Finally, theoretical and practical contributions are discussed.

\section{ONLINE FORUMS AS INFORMATION EXCHANGES}

Online forums are defined here as web-based newsgroups which allow people to post messages and comment on other messages. They generally involve some form of moderation and rules about who can post messages, initiate new «threads » and have access to all or parts of the forum. An online forum may be linked to a commercial organisation whose products are the focus of the forum, or it may be created by an individual or groups of individuals who share a common interest. Technically, online forums sit between older format bulletin boards and more modern " groups » within social media platforms such as Facebook. The focus of this paper is the forum, but many of the principles discussed here could also apply to the older and newer technologies.

Online forums can be conceptualised as network graphs consisting of nodes (human actors) and edges (social relationships), and imply structures marked by the emergence of hubs (giant component) (Dorogovtsev and Mendesy, 2002;Albert and Barabási, 2002; Newman,2003) which are influenced by mass voluntary collaborations (knowledge contributions) and interactions among members (message exchanges)(Wasco et al., 2009; Westland, 2010).

Two research streams have emerged to analyse online networks. A first stream has taken a physical sciences perspective to analyse the dynamic structure of networks and emphasizes the importance of degree distribution (the fraction of numbers of edges associated with a randomly selected member in a network) for subsequent growth of the network and its resilience to random attacks (Watts and Strogaze, 1998; Albert, Jeong and Barabási, 2000; Dorogovtsev, 2001; Newman, Strogatz and Watts, 2001). A second stream has seen online networks as extensively created communities of individuals having a shared interest from very specialized niche interests, such as historic steam railways to much larger general interest communities, characterised by knowledge collaboration (O'Reilly, 2005; Wasco et al., 2009). In other words, the conditions for an online forum being successful rely on the extension of size as well as ongoing knowledge production.

One interesting result generated from prior research leads to the phenomena of phase transition which can be understood as a tipping point at which the growth pattern of a network shows a discontinuity. In this respect, "giant components" or "hubs" play an important role in predicting phase transitions within networks . Giant components (or "hubs") comprise individuals with a high number of connections within the network (Albert et al., 2000; Dorogovtsev, 2001; Newman et al., 2001; Westland, 2010). In the theory of percolation as well as selforganised criticality (Erdös and Rényi, 1960; Bak, Tang and Wiesenfeld, 1987; Molloy and Reed, 1995; Callway, Newman and Strogats, 2000) the nature of phase transitions can facilitate a continuous and stable growth of the system, or self-sustainability in terms of the expansion of a network.

Prior research has mainly focused on either understanding the function or dynamic characters of online networks, which explains conditions for the presence of a giant component, or the importance of knowledge exchanges among members in the extension of online forums. However, there are at least two gaps in previous research. Firstly, why do members contribute knowledge online and what motivates them to do so? Secondly, how does the structure of a network influence members' contribution behaviours? Studies on the antecedents of voluntary mass contribution online are rare (Wasco et al., 2009) although their presence may provide intrinsic reasons for the 
existence of online networks. Very few studies (Wasco et al., 2009; Centola, 2013) have proposed Critical Mass theory (Oliver and Marwell, 1988) to understand online voluntary contribution behaviours. However, Critical Mass theory by Oliver and Marwell (1988) only indicates that initial contribution behaviour can promote mass contribution, and it does not explain how the initial contribution is influenced by the structure of a network.

Study of pilot results suggest that the logic of contribution behaviours initially presented by Oliver and Marwell (1988) is suitable for the analysis of online forums - members will contribute to a forum when their expected gain exceeds their sacrifice /cost. Secondly, the degree distribution - also known as the probability of a member with $K$ connections - follows the power law. In other words, the first condition for phase transition in online forums is satisfied. Thirdly, the "giant component" firstly presents itself when the mean numbers of the second neighbours exceed that of the first neighbours and is greater than 2. In other words, the second condition for phase transition is in agreement and the giant component is identified. Finally, according to complex network theories, contribution by a giant component which presents immediately after phase transition ensures continuous message exchanges in online networks.

\section{ONLINE INFORMATION AS "PUBLIC GOODS"}

Digital information can be considered to be a "public good". Four characteristics of public goods are nonrivalry of consumption, non-excludability, a decelerating production function and jointness of supply (Ostrom, 2000; Wasco et al., 2009). In other words, public goods are outputs of collective contribution, and all individuals are able to access public goods regardless of their own contributions (Snidal, 1979). Furthermore, public goods such as public open spaces and public radio are not used up after consumption (Samuelson, 1954). The cost of joint production of public goods is lower than would be the case if the good was produced separately for each consumer (Buchanan, 1966). A classic example is public television broadcast, can be seen by all at home, satisfying the criteria for joint production of a public good (Wasko et al., 2009). Similarly, digital online forums provide collective intelligence through online publication of information, whose usage will neither exclude nor diminish the capability of access or usage by other users who follow. Once a message is published, the cost of jointly supplying it remains the same no matter how many members read the message (Wasco et al., 2009).

The classic linear public good is illustrated through the utility function: $U_{i}=U_{i}\left[\left(E-X_{i}\right)+A^{*} P\left(\sum X_{i}\right)\right]$, where " $\mathrm{E}$ is an individual endowment of assets; $\mathrm{X}_{i}$ is the amount of this endowment contributed to provide the good, $\mathrm{A}$ is the allocation formula, and $\mathrm{P}$ is the production function. $\mathrm{A}$ is specified as $1 / \mathrm{N}$ and $0<1 / \mathrm{N}<\mathrm{P}<1$, where $\mathrm{N}$ is the number of individuals (Ostrom, 2000, p.139)". A specific characteristic of digital public goods is that they are indivisible, i.e. the information online remains the same however many times it has been read. Thus, the utility function of digital public goods is expressed as $\mathrm{U}_{i}=\mathrm{U}_{i}\left[\left(\mathrm{E}-\mathrm{X}_{\mathrm{i}}\right)+\mathrm{P}\left(\sum \mathrm{X}_{\mathrm{i}}\right)\right]$, with $0<1 / \mathrm{N}<\mathrm{P}<1$, since there is no allocation problem any longer.

One obvious problem associated with public goods is the social dilemma (Ostrom, 2000; Ostrom, 2010). According to Gintis (2007), the individual decision making model (named the rational actor model in economics) indicates that decisions are made to optimise a preference function subject to informational and material constraints. In other words, when every individual is rational and enjoys a public good for free, the public good will never be produced. Members of an online forum who benefit from this network but who wish to make little or no contribution, are called free riders.

The above analysis suggests that overcoming the public goods / social dilemma problem requires collective actions that are specifically embedded in voluntary cooperation (Ostrom, 2000; Wasko et al., 2009). Explanations for why people contribute to online forums have been derived from economic, sociological and biological perspectives. One significant contribution derives from game theory, which has been refined with models of direct and indirect reciprocity to incorporate an individual's expected return from their contribution (Axelrod \& Hamilton, 1981; Sugden, 1984; Dufwenberg et al., 2001; Groson, 2007; Imhof et al., 2007; Taylor \& Nowak; 2007; Nowak et al., 2010). Theories of altruism have sought to explain why an individual takes care directly of others' welfare at one's own cost (Becker,1974; Frohlich, 1974), However, there are different underpinning assumptions for these theories. Altruism behaviours often present in homogenous populations, while reciprocity requires conditions of simultaneous contribution (Nowak et al., 2010). These theories have not taken sufficient account of the scale of 
social networks relative to their heterogeneous population and subsequent activities in influencing contribution to a forum. It has been argued that critical mass theory (Oliver and Marwell, 1988) plays an important role in the development of online collective actions (Wasco et al., 2009) because it overcomes restraint assumptions associated with both theories of altruism as well as reciprocity.

Critical mass theory (Oliver et al., 1985; Marwell et al., 1988; Oliver and Marwell, 1988; Prahl et al., 1991) incorporates theories of individuals' rational choice into collective action (Centola, 2013) and argues that a group of initial contributors can pay the set-up cost and thereafter promote the mass contribution within a network. Online forums involve a minimum number of initial contributors. In the following sections, we will examine the concept of proportion of initial contributors in a large-scale social network embedded in the theory of critical mass.

\section{CRITICAL MASS}

Geng et al. (2004), describing e-communities as a type of online forum noted that they are made up of a dynamic-continuous inflow and outflow of members, who have imperfect information and memory. This aspect of dynamic e-communities calls for theories explaining how digital public goods could be contributed through the collective actions of a large, heterogeneous population. Furthermore, theories suitable for digital public goods deal with multiple processes rather than simultaneous contribution. That is, traditional theories of altruism and reciprocity alone are not able to explain mass collective actions in the emerging phenomena. Most recently, Wasko et al (2009) and Westland (2010) suggest that the theory of critical mass is relevant for understanding the digital public goods problem and free rider issue.

Critical mass theory (Oliver et al., 1985) explains how a small number of selected individuals can have a powerful, positive impact on mass collective production. Similar to threshold models (Granovetter, 1978), it focuses on the number or proportion of self-interested contributors for whom net benefit exceeds net cost. This transformation is analysed through the contagion model in biology (Dodds \& Watts, 2004), and self-organised criticality in Physics (Per Bak, Chao Tang and Kurt Wiesenfeld, 1987). In social life, one simple example is "fashion", where several selected celebrities can evoke uniform massive behaviours.

Critical mass theory is the most compelling argument of Olson's (1965) logic of collective action (Oliver and Marwell, 2001). Olson (1965) points out that a rational individual will not behave cooperatively in order to achieve their common or general interest, without incentive or punishment mechanisms that reward co-operators or punish non-co-operators. Marwell and Oliver (1993) argue that initial contributors can create positive incentives for subsequent actors, which generates a widespread activity over the group to support the production of public goods. According to Marwell and Oliver (1993), there is a possible self-reinforcing system in collective actions and it is the initial contributors who pay the set-up cost and promote future contribution behaviours of subsequence actors.

The original critical mass model developed by Marwell et al. (1988) can be employed to illustrate individuals' decisions about contributing to public goods as follows:

$\mathrm{G}=\mathrm{p}\left(\sum \mathrm{r}\right) \mathrm{I}-\mathrm{r}$,

where $G$ represents an individual's net gain from contribution. It interprets the relationship between an individual and the group in general, thus, it omits the interactions between individuals but highlights the general exchange pattern; $\mathrm{p}\left(\sum \mathrm{r}\right)$ refers to the production function of the total contribution by all parties to public goods, which specifies the relationship between inputs of total resource contribution and outputs of levels of public goods. Furthermore, the production function in this model is a $u$-concave ${ }^{1}$ or accelerating function, which facilitates increasing marginal returns. In online discussion, for instance, one response to a seed message may tell $10 \%$ of the "truth", the second one contributes to $20 \%$, the third one goes to $50 \%$, and the fourth perhaps up to $90 \%$. In other words, an accelerating production function encourages individuals to make sequential contributions that are

\footnotetext{
${ }^{1}$ Contrast to u-concave or accelerating production function, u-convex or decelerating production function that traditionally studied in economical models fostered initial actions, which leads to strategic action and free-ride problem (Oliver and Marwell, 2001). For a given public good, the benefit to individual A exceeds largely A's cost, thus it worth A to contribute this public good. Individual B knows A will whatever contribute, B could pay little or nothing but enjoy this public good in the future.
} 
embedded in previous outputs, because additional contributions could accelerate achievement of certainty. However, the central challenge is to start collective actions because rational individuals will contribute in the late stage in order to enjoy higher payoffs; I is an individual's interest level in the public good; And r means an individual's contribution resource. That is, when $\mathrm{p}\left(\sum \mathrm{r}\right)>\mathrm{r} / \mathrm{I}$, .i.e. the total payoff from all contributions to public goods exceeds the individual's $\mathrm{r} / \mathrm{I}$ ratio, an individual will make a positive contribution decision. In other words, the value of a given public good is subjected to available resources and the willingness to pay: the higher the interest level, the more possible that an individual contributes; the richer the resources available, the bigger the outputs.

It can be concluded that there are two important assumptions in the critical mass model: the accelerating production function that highlights the feasibility problem ${ }^{2}$, and the group heterogeneity that allows either highly interested or resourceful individuals to pay the early start up cost of collective actions. The idea of critical mass is related to exactly these kinds of contributors. In this sense, the critical mass members attract numerous others to contribute sequentially. From this, we derive our first proposition:

Proposition 1: Critical mass is related to individual members' motivation to voluntarily share knowledge online.

The literature cited above indicates that the theory of critical mass contribution describes a phenomena of phase transition that can finally lead to stable and dynamic collective collaboration. However, the model proposed originally by Oliver and Marwell's (1988) does not discuss how to identify the critical mass members in groups. This could be one reason why theories of critical mass are often surrounded by disagreements. The following sections will discuss how the initial critical mass contributors are identified and embedded in network analysis.

\section{OVERVIEW OF ONLINE NETWORKS AND CRITICAL MASS MEMBERS}

Online social network is a topic in scale-free complex networks (Wang, Li and Chen, 2006; Wang and Dai, 2009; Shi, 2011). The concept of scale-free is developed by Barabási and Albert (1999) ("BA model") from the random network theory (Erdös and Rényi, 1960) ("ER" model" and the Small World Network (Watts and Strogatz, 1988) ("WS" model).

In the random network theory (ER), a member chooses to randomly connect to another in a fixed $\mathrm{N}$ numbers of members in a community with a given probability $\mathrm{p}$. As a result, the network contains on average p*N(N-1)/2 edges. This is a typical binomial problem, members either connect or not to each other. That is, the probability which a member can have $\mathrm{k}$ connections $\mathrm{Pk}$ can be described as $P_{k}=\left(\begin{array}{c}n-1 \\ k\end{array}\right) p^{k}(1-p)^{n-1-k}$, the average connections is therefore $\bar{K}=p(N-1)$. For a large $\mathrm{N}$, the degree distribution follows the Poisson distribution: $P_{K}=e^{-\bar{k}} \bar{k}^{k} / k$ !. In the qualitative interpretation, the Poisson form indicates the vast majority of members have roughly the same connections and a few members have connections which deviate significantly from the average.

The main criticism of the random network theory approach is missing reference to preferential attachment (Watts and Strogatz, 1988; Chakrabarti and Faloutsos, 2006). In the real world, members typically choose to connect with members who are close to them rather than randomly. Stimulated by this observation, Watts and Strogatz (1988) suggest the Small World Model (WS), which starts with N nodes set in a circle and each is connected to its two neighbours by their sides. Thereafter, a probability $\mathrm{p}$ is given to members to form new edges or connections. The new edges are chosen equally at random. As a result, the degree distribution is similar to the bell curves of random networks with the peak at $\bar{K}$ but with a shorter distance among members.

Both the Erdös-Rényi and Watts-Strogatz models share an assumption that there are fixed $\mathrm{N}$ members who are either randomly connected or reconnected. However, as Barabasi and Albert (1999) point out, a real world network can enjoy a continuous increasing number of members throughout its lifetime. Combining the ER and WS model, they collect data from websites and found that the degree distribution obeys a power law. Different from the Poisson distribution, the Power law distribution indicates the absence of the mean connections (a peak in the bell

\footnotetext{
${ }^{2}$ Feasibity problem refers to finding an initial point which satisfys all constraints in an algorithm problem. In our case, it is associated with uconcave production function.
} 
curve) but the occurrence of hubs (infinitely close to the $\mathrm{X}$ axis). In other words, several hubs have the majority connections but the majority only have few or no connectivity with others in real networks such as social networks. According to Barabasi and Albert (1999), there is a continuous hierarchic variation but no typical scale connectivity between members, and this is a so called scale-free network.

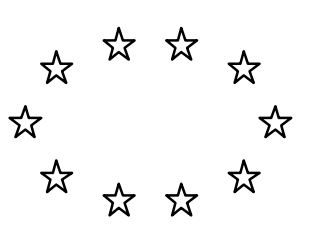

$\mathrm{P}=0$, ten isolated nodes

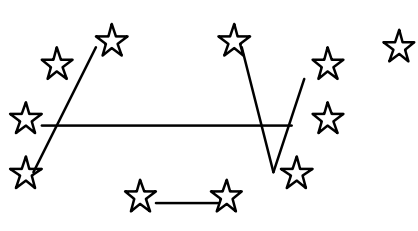

$\mathrm{P}=1 / \mathrm{N}=0.1$

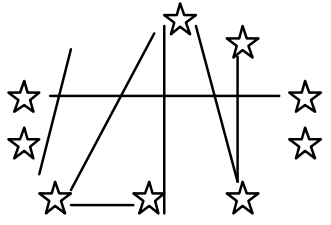

$\mathrm{P}=15 / \mathrm{N}=0.15$

Source: Erdös P. Rényi A.(1960). On the evolution of random graphs. Publ. Math. Inst. Hung. Acad. Sci., 5, 17-60.)

Figure 1. An Illustration Of The Evolution Of Random Networks

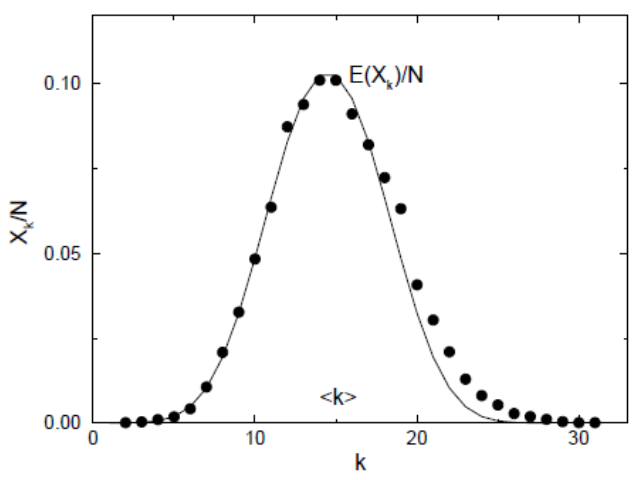

Source: Newman MEJ. (2003), The structure and function of complex networks, SIAM Review, 45, 167-256,

Figure 2. An Example Of The Bell Curve Of Random And Small World Networks

\section{Scale-free Networks}

The internet and increasing computing power facilitate data collection in large size networks. Recently, empirical research in complex networks such as Web hyperlinks, protein networks, wifi networks, actor cooperation networks etc suggests the power law tail charactering the degree distribution (Barabasi and Albert, 1999; Newman,2001; Dorogovtsev, 2002; He, Liu et Wang, 2009; Wang and Dai, 2009; Shi, 2011). In order to illustrate the evolution of scale-free networks, Barabasi and Albert (1999) address the BA model focusing on two generic attributes in many real world networks, exhibited through growth and preferential attachment. For example, the citations of an academic paper grow exponentially in time through new citation, and the new citation is more likely to depend on the cited times which may indicate the popularity of acceptance. These two properties demonstrate a good example of simplifying complex phenomena.

The introduction to the logic of the BA model that has a power law degree distribution is as follows:

Growth: starting with a small number $m_{0}$ of nodes, and a new node is introduced at every time step to $m$ existed nodes, with $m \leq m_{0}$, in other words, this new node has m edges.

Preferential Attachment: the probability $\Pi$ that this new node will connect an existing node $_{i}$ depends on the degree $k_{i}$ of node $_{i}$ and $k_{j}$ of existed node ${ }_{j}$, so that, $\prod_{i}=\frac{k_{i}}{\sum_{j} k_{j}}$ 
After $t$ time, there are $\mathrm{N}=\mathrm{t}+m_{0}$ nodes and $E=m_{0}\left(m_{o}-1\right) / 2+m t$ edges, and the simulation by Barabasi and Albert (1999) indicates that a network evolves into the state where few highly connected hubs can dominate the network's connectivity. In other words, the network is more resilient to random attacks than particular attacks on hubs. A mathematical relationship is introduced to describe the degree distribution in scale-free networks: $P(k) \sim k^{-\gamma}$ where $P(k)$ stands for the degree distribution of the probability of nodes connecting to $\mathrm{k}$ others members, and $Y$ is the degree exponent which is equals to 3.

The interesting result emerging from this simulation is that the exponent is independent from $m$ which is the only parameter in the model (Barabasi and Albert,1999). In other words, the growth of networks is mostly influenced by the hubs which dictate the connectivity of the network.
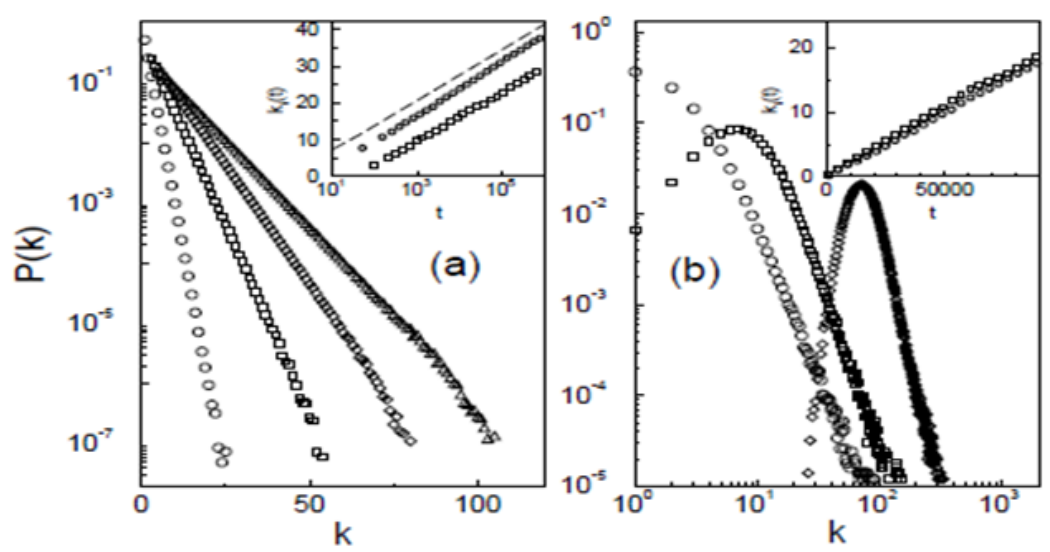

(a) Degree distribution for model $\mathrm{A}$ for $m_{0}=\mathrm{m}=1$ (circles), $m_{0}=\mathrm{m}=3$ (squares), $m_{0}=\mathrm{m}=5$ (diamonds), and $m_{0}=\mathrm{m}=7$ (triangles). The size of the network is $\mathrm{N}=800,000$. Inset: time evolution for the degree of two vertices added to the system at $t_{1}$ $=7$ and $t_{1}=97$. Here $m_{0}=\mathrm{m}=3$. The dashed line follows $k_{i}(t)=m \ln \left(m_{0}+t-1\right)$. (b) The degree distribution for model B for $\mathrm{N}$ $=10,000$ and $\mathrm{t}=\mathrm{N}$ (circles), $\mathrm{t}=5 \mathrm{~N}$ (squares), and $\mathrm{t}=40 \mathrm{~N}$ (diamonds). Insert: time dependence of the degree of two vertices. The system size is $\mathrm{N}=10,000$. After Barabasi, Albert and Jeong (1999).

Figure 3.

\section{Dynamic Processing In Scale-Free Networks - Highest Degree}

As discussed above, hubs reflect the connectivity that a real network allows. A network is vulnerable by deletion of its highest-degree members and their associated edges but resilient to random attacks (Albert et al., 1998; Broder et al., Jeong et al.; Newman, 2003). In other words, if there is one path linking one member to the critical components, then one member can communicate with an extensive fraction of the entire network. If the path is deleted or the associated critical component is removed, one member can only communicate with a few others at most (Newman, 2003).

Empirical studies (Dorogovtsev and Samukhin, 2001; Moreira et al., 2002) on the highest or maximum degree in scale-free networks suggest that, if $p_{k} \sim k^{-a}$, then $k_{\max } \sim n^{1 /(a-1)}$. For instance, a central vertex in a scalefree network of 1,000 vertices with the exponent $a=3$ can have 10 maximum connections, and 1,000 maximum connections with the exponent $a=2$. In other words, the number of edges has a negative relationship with the control parameter $a$. According to Albert et al. (2000), there is a critical value for $a=2$ at which the critical component occurs, and below which the networks can be completely connected. Albert et al. (2000) further found that $a$ between 2-3 is in agreement with real Internet based networks such as online forums.

Furthermore, if the parameter $a$ is known, noting for example $a=3$, while the size of networks increases 
from 1,000 to 10,000 members, the maximum connections increase from 10 to 100 . The maximum degree is therefore positively associated with the size of networks. However, as a network is evolving with time and new nodes are continuously joining into the networks, the size can tend towards infinity, which makes it difficult to compute the maximum degree. In most cases, one can work with the average of maximum degree (Albert et al. 2000). Mo'ri (2005) later proposed an alternative method which indicates that $K_{\max } \sim t^{1 / 2} u$. Where $u$ is a set of absolutely continuous variables, following a normal distribution. This result is generated from the condition when every new member is attributed with only one edge. Zhou, Ye and Yan (2010) further propose the experimental formula for computing the average of maximum degrees in infinitive BA networks: $E\left[k_{m}(t)\right] \approx(20.11 m+329.8) t^{1 / 2}$, where $m$ represents the number of edges uniformly associated to a new member $(1 \leq m<3), t$ is number of periods of evolution. When $m=3, \ln u=\ln \left[t^{-1 / 2} k_{m}(t)\right] \sim N\left(0.4884,0.0745^{2}\right)$.

However, it is not difficult to compute the highest degree in real world scale free networks which always have a finite size. Nevertheless, there may be more than one member with maximum connections, and those members have been referred to as a giant component or hubs. The important position of the giant component in networks will be further discussed in the following sections.

\section{Self-Organised Criticality}

The discovery of scale-free networks where degree distributions obey the power law form has evoked great interest in network research. In fact, the emergence of complex network theory is a topic about the complex system which has been studied in diverse fields from biology, physics to economy (Wang et al. ;2006). To date, there is no widely accepted definition of a complex system. However, the shared concept in this field leads to the theory of selforganised criticality (SOC)( P. Bak, C. Tang \& K. Wisenfeld, 1987) which explains how the non-linear interactions among mass nodes and in particular in hubs can bring a phase transfer even under conditions of non central control, asymmetric information and local movement. This is often employed to explain the disappearance of the dinosaur, avalanches, earthquakes and bull or bear stock markets (Wang \& Dai, 2009).

Self-organised criticality is considered as a 'must' connection to scale-free networks which is further supported by the observation of the power law tails (He et al., 2009). The Sandal model by Bak et al. (1987) is the classical example to illustrate the concept of SOC. Consider a desk where a number of grains is introduced randomly. Thereafter a new grain is added and the height of sandal increases by repeating this step. As the pile grows, its slope becomes steeper until additional grain triggers a local avalanche. After a while, the pile reaches a critical height $H i$, newly joined grain leads to a large avalanche and the pile spreads aside. A critical point occurs where a single movement such as falling of a new grain at a tipping point can evoke the change of the whole system. This is self-organised in that there is no invisible hand or external factors which are manipulating this phase transfer, because this pattern occurs spontaneously.

Results of extensive simulations on the sandal model (Albert and Barabasi, 2000) indicate that the distribution of the size of the avalanche follows the power law form and is described as : $N(s)=s^{-t}$, where $\mathrm{N}(\mathrm{s})$ stands for the size of collapse event, $t$ is the exponent parameter. The negative of $t$ indicates that the size of collapse decreases when the size of sandal $S$ increases.

For both scale-free as well as self-organised criticality theory, the critical or giant component is widely recognized as the key factor of interest. In the theory of scale-free network, giant component or hubs are vertexes which own the majority connections, and this aspect is crucial against random attack to the network. That is, if hubs are not attacked, the network remains largely undamaged. In the theory of self-organised criticality, the critical component predicts the phase transaction. The idea is that local disturbances in time and space are accumulated exponentially, which allows the occurrence of the giant component at the critical point over which the avalanche is generated. 


\section{“Giant Component"}

The motivation behind self-organised criticality theory is that it can show phase transitions on scale free networks. In fact, there are extensive studies about phase transitions on random networks embedded in the bond percolation theory (Erdös and Rényi, 1960; Burton and Keane, 1989; Molly and Reed, 1995).

Previous numerical analytical results (Albert and Barabasi, 2000) derived from percolation process ${ }^{3}$ in random networks with Poisson degree distribution indicates that the condition for the emergence of the critical component is: $p_{c=} \frac{1}{z-1}$, where $p_{c}$ is the critical point when the giant component is emerging; $z(=3)$ is the maximum acquaintance of the origin site; z-1 means that at least one of z-1 edges connects the origin node to others. Similar to the theory of self-organised criticality which describes the phase transition happening almost immediately after the occurrence of the critical components, linkage possibility $p \geq p_{c}$ assures that network growth is continuous or selfsustainable since the giant component appears and they own infinitive outgoing edges.

Results from studies on Power law degree distribution networks show a qualitatively similar phase transition behaviour to that of Poisson random graph (Albert et al., 2000; Newman et al., 2000; Broder et al., 2001). For instance, the configuration model (Newman et al., 2001; Newman, 2003) is a general approach to random graphs with power law degree distribution. According to this model, there is a set of $n$ value of degree sequence $\left\{k_{1}, k_{2},,,,, k_{n}\right\}$. If one chose randomly an edge (node which is reachable at one side belongs to $G_{0}(x)$ ), one can always find a vertex plus some other cluster of vertices at the other end of this edge (noted as $G_{1}(x)$ ). Nodes are thereafter classified into two groups and the two proceed in generating functions of the degree distributions: $G_{0}(x)=\sum_{k=0}^{\infty} P(k) x^{k}, G_{1}(x)=\frac{\sum_{k} k P(k) x^{k-1}}{\sum_{k} k P(k)}=\frac{1}{\langle k\rangle} G_{0}^{\prime}(x)$, where $\langle k\rangle$ is the average number of the first neighbours of a node or the mean degree in the network $G_{0}^{\prime}(1) . k P(k)$ tells that a randomly chosen edge associated to a node with $k$ degree is proportional to the degree of that node. $x^{k}$ means there are $k$ ways to reach a node with degree $k$. Given an example by Newman et al., (2001), there are 1000 members in a finite network, and each of them has acquaintances range from zero to five, $\{86,150,363,238,109,54\}$, therefore, $G_{0}(x)=\frac{86+150 x+363 x^{2}+238 x^{3}+109 x^{4}+54 x^{5}}{1000}$; $\langle k\rangle=\sum_{k} k p_{k}=0+1 * \frac{150}{1000}+2 * \frac{363}{1000}+3 * \frac{238}{1000}+4 * \frac{109}{1000}+5 * \frac{54}{1000}=2.296 \approx 2 ;$

Following the outside edges of the first neighbours, there are next-nearest neighbours and this process is repeated until the end of those edges. To distinguish from the first selected edge, Newman (2003) introduces the concept of excess degree to depict those leaving edges.

With distribution probability, the generating function for the average cluster size of first and the second neighbours can be defined because the degree distribution probability of one vertex is the fraction of the total number of degrees: $\langle s\rangle=1+\frac{G_{0}^{\prime}(1)}{G_{1}^{\prime}(1)}=1+\frac{z_{1}^{2}}{z_{1}-z_{2}}$, where $G_{0}^{\prime}(1)=\langle k\rangle=z_{1}$, and $z_{2}=\left\langle k^{2}\right\rangle-\langle k\rangle=G_{0}^{\prime}(1) G_{1}^{\prime}(1)$, the average number of second neighbours. It shows that the condition for phase transitions in scale-free networks can be written as: $\sum_{k} k(k-2) P(k)=0$ or $\frac{\left\langle k^{2}\right\rangle}{\langle k\rangle}=2$, and $\left\langle k^{2}\right\rangle=\sum_{k} k^{2} P(k)=\langle k\rangle^{2}+\langle k\rangle$. In other words, the giant component presents when the average number of the second neighbours exceeds the average number of the first neighbours (Dorogovtsev and Mendes, 2001). This result is interesting because it suggests that the Scale Free networks with exponent parameter $a$

\footnotetext{
${ }^{3}$ Results are generated from percolation process on a Cayley tree with coordination number $z=3$, and average degree approaches to 2 . A Cayley tree holds a property in common with radaom graph (Albert and Barabasi, Statistical mechanics of complex networks, 2002).
} 
between 2 and 3 always percolates, indicating that critical component always exists in networks, if the average degree of networks is superior to 2 (Cohen, Erez, ben-Avraham and Havlin, 2000; Albert et al., 2000; Newman, 2001). After the phase transition, the size of giant component is continuously growing, and near the critical point of transitions, the average size of the giant component is computable.

\section{PROPOSED MODEL OF CRITICAL MASS CONTRIBUTION}

As discussed previously, the original critical mass theory (Marwell and Oliver, 1988) can be applied to understand members' voluntary contribution behaviour on online forums. In order to identify critical mass members in online forums, network structural analysis should be incorporated. There exists a critical probability $p_{c}$ at which a phase transition occurs and a "giant" component with computable size firstly appears. With this condition, online forums could enjoy continuous growth in terms of size and message exchanges. In other words, the knowledge sharing within networks is dependent on giant component comprised of "critical mass members". This is in agreement with the critical mass theory (Marwell and Oliver, 1988) which highlights the importance of minimum initial contributors in evoking mass collective actions. Similar to the work of Westland (2010), we understand that 'giant' component and critical mass member have equivalent meaning regarding their importance in phase transition.

It has been widely accepted that members who participate in online knowledge sharing such as initially proposing a message and joining others' discussions will benefit from publicly available information support (Preece, 2002; Ridings and Gefen, 2004b; Wasko et al., 2009). A seed message is considered potentially useful information when it is discussed among members. The discussed message is often value-added knowledge since discussers could contribute their further understandings to the seed message. Therefore, the total number of discussed seed messages which are accessible to each member represent their benefits. This is consistent with the view of the characteristics of online knowledge as digital public goods (Wasko et al., 2009). Therefore:

Proposition 2: The benefit for critical mass members can be represented through the total number of discussed messages, which are accessible to every member.

According to Critical mass theory (Marwell and Oliver, 1988), contribution behaviours are in proportion to members' potential benefit from the public goods. Similarly, the more attractive that a particular topic appears to members of an online forum, the more possible that members would participate online discussions around that topic. Researchers such as Odlyzko and Tilly (2005) and Westland (2010) have argued that the size of a group could be a measure of the interest level of such group. We propose therefore that the size of audience of messages created by critical mass members is a good indicator of the interest level of these messages. In addition, we argue that the interest level of a message can reflect the interest level of the poster since a member publishes a message and seeks further discussions through a lengthy "thread", although it is necessary to note that members who are interested in a particular topic may not participate in discussions around that topic (Wasko et al., 2009). That is, the size of audience includes members who answer messages and those who are merely watching.

Westland (2010) introduces the logarithmical concept, $\log ($ n̆s (p)) representing the logarithm of average number of each member's special interest clusters containing s members, as the indicator of the level of interest of the online community. In our study, we further define the size of cluster S as satisfying the condition where the group of critical mass members firstly appears, $\langle s\rangle=1+\frac{G_{0}^{\prime}(1)}{G_{1}^{\prime}(1)}=1+\frac{z_{1}^{2}}{z_{1}-z_{2}}$ (Newman, 2003). The use of logarithmical measurement on humans' perception is common in biological and physical studies (He, Liu and Wang, 2009). Logarithmical measure is more robust than simple summarization of numbers of interested individuals. It is presumed that human perception logarithmically transforms intensities for obtaining a wider perceptive range that crosses multiple orders of magnitude. This idea is mostly reflected through examples such as human perception of light, sound and other sensory information (Westland, 2010).

Proposition 3: The level of critical mass members' interests in supplying knowledge online can be represented through the logarithm of the total number of replied and viewed messages created by critical mass members. 
According to Oliver and Marwell (1988), the cost of contribution is the resource used up by the contributor. de Valck et al. (2009) argues that in the context of an online community, members who participate in online discussions may encounter risks which are associated with members' private resources, such as time, psychology and social risks. For example, a devoted member contributes a lot of time participating in online discussions, which leads to less time for enjoying something else. Furthermore, members may process their private information through Internet channels, where anxieties could occur if the capacities of online communities to treat such information are questioned. Again, members may judge others' options, and their judgment may be influenced by others both positively as well as negatively. An individual who belongs to a social group is unwilling to be excluded and/or to take related social risks. However, the above mentioned resources for contribution can be represented through published messages. For instance, time cost is proportionally related with posting messages. Psychological barriers such as anxieties can be demonstrated through either continuing to debate with others or giving up discussions. Again, members may be either encouraged or discouraged to post messages according to the popularity of messages posted by them. Riding and Wasco (2010) summarize that message volume can represent contribution resources. It is therefore suggested that published messages are knowledge which reflect intellectual resources owned by message publishers.

Proposition 4: The cost of contributions by critical mass members can be represented by the total number of messages posted by them.

Finally, the production function in the critical mass model (Marwell, Olivier and Prahi, 1988) describes the relationships between input recourse of all contributors and outcomes of public goods. It is an accelerating function because successive contributions generate larger payoffs (Oliver, Marwell and Teixeira, 1985). It is true in online forums, where value-added messages not only benefit for members but also for visitors.

Proposition 5: The production function of online forums can be represented through the total number of posts by reply and by view.

\section{MANAGERIAL IMPLICATIONS}

Online forums play an increasingly important role in business strategy, as communication channels between companies and their customers have been transformed from predominantly bilateral to increasingly multilateral. They allow customers to become "co-creators" of value and provide a practical application of this aspect of service dominant logic (Vargo and Lusch, 2008a). This "co-creation of value") presumes a willingness and ability of fellow consumers to sacrifice time and effort to produce benefits which may be appropriated by others, and not necessarily reciprocated (Wasco, Teigland and Faraj, 2009).

The literature cited above indicates that critical mass contribution models describe a transition model that leads to stable and dynamic collective collaboration. However, Oliver and Marwell's (1988) model does not analyse the ratio of initial contributors and their identification in groups. This could be one reason why theories of critical mass are often surrounded by disagreements.

Companies providing online forums should address the phenomena of phase transition which can be understood as a tipping point at which the growth pattern of a network shows a discontinuity. "Giant components" or "hubs" play an important role in predicting this phase transitions within networks . Hosts of online forums should pay strong attention to these giant components or hubs, which comprise individuals with a high number of connections within the network

Within any model, an online forum must understand the logic of contribution behaviours. Essentially, members will contribute to a forum when their expected gain exceeds their sacrifice /cost ad explanations for such contributions have been derived from economic, sociological and biological perspectives.

Forum hosts should not rely on altruism alone to encourage contributions.. Altruism behaviours often present in homogenous populations, whereas reciprocity requires conditions of simultaneous contribution among 
often heterogeneous groups. Theories of altruism have not taken sufficient account of the scale of social networks relative to their heterogeneous population and subsequent activities in influencing contribution to a forum. In this respect, critical mass theory provides firms with a useful framework for understanding the development of online collective actions, by incorporating theories of individuals' rational choice into collective action. It is based on the principal that a group of initial contributors can pay the set-up cost and thereafter promote the mass contribution within a network. Critical mass theory explains how a small number of selected individuals can have a powerful, positive impact on mass collective production, by focusing on the number or proportion of self-interested contributors for whom net benefit exceeds their net cost.

Companies have traditionally sought to recruit opinion leaders to be initial adopters of new products, in the expectation that hey will pass on word of mouth recommendation to others. Recruitment to online forums can usefully adopt similar principles and a critical mass model can help to identify and quantify the number of such "seedcorn" members who are needed in order to pass a tipping point at which the forum becomes sustainable. The model offers a big advantage over random network models, because in the real world, members typically choose to connect with members who are close to them rather than randomly with others.

\section{AUTHOR INFORMATION}

Qunying Huo is a doctoral candidate in Marketing of the Open University's Associated Research Centre at ESC Rennes. Her research interests involve with social computing. Her works has been published in Journal of Marketing Management. Email: qunying.huo@esc-rennes.fr

Adrian Palmer is Professor of Marketing at ESC Rennes, France. His first career was in services marketing and management. Since joining academia, he has researched and published extensively on the subject of services buyer behaviour. His book "Principles of Services Marketing", now in its seventh edition, is widely used throughout the world to provide a grounding in the challenges and opportunities of marketing services. His research has been published in Journal of Marketing Management, European Journal of Marketing and Harvard Business Review. Email: mail@apalmer.com (corresponding author)

\section{REFERENCES}

Albert, R., \& Barabási, A. L. (2002). Statistical mechanics of complex networks. Reviews of modern physics, 74(1), 47.

Albert, R., Jeong, H., \& Barabási, A. L. (2000). Error and attack tolerance of complex networks. Nature, 406(6794), $378-382$.

Alger, I. (2010). Public Goods Games, Altruism, and Evolution. Journal of Public Economic Theory. July, 12(4), $789-813$.

Axelrod, R., \& Hamilton, W. D. (1981). The evolution of cooperation. Science, 211(4489), 1390-1396.

Bak, P., Tang, C., \& Wiesenfeld, K. (1987). Self-organized criticality: An explanation of 1/f noise. Physical Review Letters, $59(4), 381-384$.

Barabási, A. L., \& Albert, R. (1999). Emergence of scaling in random networks. science, 286(5439), 509-512.

Becker,Gary S. (1974). A Theory of Social Interactions. Journal of Political Economy. 82(6), 1063-1093.

Bimber, B., Flanagin, A. J., \& Stohl, C. (2005). Reconceptualizing collective action in the contemporary media environment. Communication Theory, 15(4), 365-388.

Boccaletti, S., Latora, V., Moreno, Y., Chavez, M., \& Hwang, D. U. (2006). Complex networks: Structure and dynamics. Physics reports, 424(4), 175-308.

Broder, A. Z., Glassman, S. C., Manasse, M. S., \& Zweig, G. (1997). Syntactic clustering of the web. Computer Networks and ISDN Systems, 29(8), 1157-1166.

Buchanan, J.M. (1966). Joint supply, externality and optimality. Economica, 33(132), November, 404-415.

Butts, C. T., \& Cross, B. R. (2009). Change and external events in computer-mediated citation networks: English language weblogs and the 2004 US electoral cycle. Journal of social structure, 10(3).

Callaway, D. S., Newman, M. E., Strogatz, S. H., \& Watts, D. J. (2000). Network robustness and fragility: Percolation on random graphs. Physical review letters, 85(25), 5468.

Centola, D. (2008). Failure in complex social networks. The Journal of Mathematical Sociology, 33(1), 64-68.

Centola, D. M. (2013). Homophily, networks, and critical mass: Solving the start-up problem in large group collective action. Rationality and Society, 25(1), 3-40.

Clauset, A., Shalizi, C. R., \& Newman, M. E. (2009). Power-law distributions in empirical data. SIAM review, $51(4), 661-703$.

Chakrabarti, D., \& Faloutsos, C. (2006). Graph mining: Laws, generators, and algorithms. ACM Computing Surveys (CSUR), $38(1), 2$.

Cohen, R., Ben-Avraham, D., \& Havlin, S. (2002). Percolation critical exponents in scale-free networks. Physical Review E, 
$66(3), 036113$.

Cohen, R., Erez, K., Ben-Avraham, D., \& Havlin, S. (2000). Resilience of the Internet to random breakdowns. Physical review letters, 85(21), 4626.

Dawkins, R. (1989). The selfish gene. 1976. revised edn. Oxford.

Demange, E., Henry, E., \& Préau, M. From collaborative research to community-based research.

De Valck, K., Van Bruggen, G. H., \& Wierenga, B. (2009). Virtual communities: A marketing perspective. Decision Support Systems, 47(3), 185-203.

Dholakia, U. M., Kahn, B. E., Reeves, R., Rindfleisch, A., Stewart, D., \& Taylor, E. (2010). Consumer behavior in a multichannel, multimedia retailing environment. Journal of Interactive Marketing, 24(2), 86-95.

Dodds, P. S., \& Watts, D. J. (2004). Universal behavior in a generalized model of contagion. Physical review letters, 92(21), 218701.

Dorogovtsevyz, S. N., \& Mendesy, J. F. F. (2002). Evolution of networks. Advances in Physics, 51(4), 1079-1187.

Dorogovtsev, S. N., Mendes, J. F. F., \& Samukhin, A. N. (2001). Size-dependent degree distribution of a scale-free growing network. Physical Review E, 63(6), 062101.

Dufwenberg, M., U. Gneezy, W. Güth, \& E. Van Damme (2001). Direct vs Indirect Reciprocity: An Experiment. Homo Oecon. $18,19-30$.

Edwards, W. K., Newman, M. W., Sedivy, J. Z., \& Smith, T. F. (2009). Experiences with recombinant computing: Exploring ad hoc interoperability in evolving digital networks. ACM Transactions on Computer-Human Interaction (TOCHI), 16(1), 3.

Ellison, N. B. (2007). Social network sites: Definition, history, and scholarship. Journal of Computer-Mediated Communication, 13(1), 210-230.

Erdös, P., \& Rényi, A. (1960). Additive properties of random sequences of positive integers. Acta Arithmetica, 6(1), 83-110.

Flanagin, A. J., Flanagin, C., \& Flanagin, J. (2010). Technical code and the social construction of the internet. new media \& society, 12(2), 179-196.

Füller, J., Matzler, K., \& Hoppe, M. (2008). Brand community members as a source of innovation. Journal of Product Innovation Management, 25(6), 608-619.

Fortunato, S. (2010). Community detection in graphs. Physics Reports, 486(3), 75-174.

Frigg, R. (2003). Self-organised criticality - what it is and what it isn't. Studies In History and Philosophy of Science Part A, 34(3), 613-632.

Frohlich, N. (1974). Self-Interest or Altruism, What Difference?. The Journal of Conflict Resolution. March, 18(1), 55-73.

Gintis, H. (2007). A framework for the unification of the behavioral sciences. Behavioral and brain sciences, 30(1), 1-15.

Grabowski, A., \& Kosinski, R. A. (2010). Percolation in real on-line networks. Acta Physica Polonica B, 41(5), 1135.

Granovetter, M. (1978). Threshold models of collective behavior. American journal of sociology, 1420-1443.

Groson, Rachel T.A. (2007). Theories of Commitment, Altruism and Reciprocity: Evidence From Linear Public Goods Games. Economic Inquiry. April, 45(2), 199-216.

Imhof, Lorens A., Drew Fudenberg and Martin A. Nowak. (2007). Tit-for-tat or Win-stay, Lose-shift?. Journal of Theoretical Biology. 247, 574-580.

Hamilton, W. D. (1964). The genetical evolution of social behaviour. II. Journal of theoretical biology, 7(1), 17-52.

Harris, L., \& Rae, A. (2009). Social networks: the future of marketing for small business. Journal of business strategy, 30(5), $24-$ 31.

He, W., Qiao, Q., \& Wei, K. K. (2009). Social relationship and its role in knowledge management systems usage. Information \& Management, 46(3), 175-180.

Hinrichsen, H. (2000). Non-equilibrium critical phenomena and phase transitions into absorbing states. Advances in physics, 49(7), 815-958.

Jeong, H., Mason, S. P., Barabási, A. L., \& Oltvai, Z. N. (2001). Lethality and centrality in protein networks. Nature, 411(6833), 41-42.

Levy, M. (2009). Web 2.0 implications on knowledge management. Journal of Knowledge Management, 13 (1), $120-134$.

Marwell, G., Oliver, P. E., \& Prahl, R. (1988). Social networks and collective action: A theory of the critical mass. III. American Journal of Sociology, 502-534.

Meisel, C., \& Gross, T. (2009). Adaptive self-organization in a realistic neural network model. Physical Review E, 80(6), 061917.

Molloy, M., \& Reed, B. (1995). A critical point for random graphs with a given degree sequence. Random structures \& algorithms, 6(2-3), 161-180.

Molloy, M., \& Reed, B. (1998). The size of the giant component of a random graph with a given degree sequence. Combinatorics probability and computing, 7(3), 295-305.

Moreira, A. A., Andrade Jr, J. S., \& Amaral, L. A. N. (2002). Extremum statistics in scale-free network models. Physical review letters, 89(26), 268703.

Móri, T. F. (2005). The maximum degree of the Barabási-Albert random tree. Combinatorics, Probability and Computing, 14(03), 339-348.

Newman, M. E. (2003). The structure and function of complex networks. SIAM review, 45(2), 167-256.

Newman, M. E., Strogatz, S. H., \& Watts, D. J. (2001). Random graphs with arbitrary degree distributions and their applications. 
Physical Review E, 64(2), 026118.

Nowak, Martin A., Corina E. Tarnita and Tibor Antal. (2010). Evolutionary Dynamics in Structured Populations. Philosophical Transaction of The Royal Society B. 365, 19-30.

Nowak, M. A., \& Roch, S. (2007). Upstream reciprocity and the evolution of gratitude. Proceedings of the Royal Society B: Biological Sciences, 274(1610), 605-610.

Oliver, Pamela E. 1980. Rewards and Punishments as Selective Incentives for Collective Action: Theoretical Investigations. American Journal of Sociology. 85, 1356-1375.

Oliver, P., Marwell, G., \& Teixeira, R. (1985). A theory of the critical mass. I. Interdependence, group heterogeneity, and the production of collective action. American journal of Sociology, 522-556.

Oliver, P. E., \& Marwell, G. (1988). The Paradox of Group Size in Collective Action: A Theory of the Critical Mass. II. American Sociological Review, 1-8.

Oliver, P. E., \& Marwell, G. (2001). Whatever Happened to Critical Mass Theory? A Retrospective and Assessment. Sociological Theory. November, 19(3), 292-311.

Olson, Norman C. Attitude control system for vtol aircraft. U.S. Patent No 3,181,810, 4 mai 1965.

O'reilly, T. (2005). What is web 2.0.

Ostrom, E. (2000). Collective action and the evolution of social norms. The Journal of Economic Perspectives, 14(3), 137-158.

Ostrom, E. (2010). Polycentric systems for coping with collective action and global environmental change. Global Environmental Change, 20(4), 550-557.

Payne, A., Storbacka, K., Frow, P. \& Knox, S. (2009). Co-creating brands: diagnosing and designing the relationship experience. Journal of Business Research, 62, 379-389.

Prahl, R., Marwell, G., \& Oliver, P. E. (1991). Reach and selectivity as strategies of recruitment for collective action: A theory of the critical mass, V*. Journal of Mathematical Sociology, 16(2), 137-164.

Preece, J. R. (2007). SHARP (2002): Interaction Design: Beyond Human-Computer Interaction. Crawfordsville: John Wiley and Sons, Inc. Answers. com Technology.

Reichardt, J., \& Bornholdt, S. (2006). Statistical mechanics of community detection. Physical Review E, 74(1), 016110.

Ridings, C. M., \& Gefen, D. (2004). Virtual community attraction: Why people hang out online. Journal of Computer-Mediated Communication, 10(1), 00-00.

Ridings, C., \& Wasko, M. M. (2010). Online discussion group sustainability: Investigating the interplay between structural dynamics and social dynamics over time. Journal of the Association for Information Systems, 11(2), 1.

Samuelson, P. A. (1954). The pure theory of public expenditure. The review of economics and statistics, 36(4), $387-389$.

Sanders, A. K. \& File, P. C. (2011), Giving Users A Plain Deal: Contract-Related Media Liability For Unmasking Anonymous Commenters. Comm. L. \& Pol'y, 16(2), 197-226.

Shi, D.H.(2011), Theory of network degree distributions (M), Beijin, Higher Education Press.

Snidal, D. (1979). Public goods, property rights, and political organizations. International Studies Quarterly, 532-566.

Sugden, R. (1984). Reciprocity: the supply of public goods through voluntary contributions. The Economic Journal, 94(376), $772-787$.

Taylor, Christine and Martin A. Nowak, (2007). Transforming the Dilemma. Evolution. October, 61(10), 2281-2292.

Vargo, S.L. \& Lusch, R.F.(2008a), Service-dominant logic: Continuing the evolution. Journal of the Academy of Marketing Science. 36 (1), 1-10.

Xiao, W. J., Chen, W. D., \& Parhami, B. (2011). On necessary conditions for scale-freedom in complex networks, with applications to computer communication systems. International Journal of Systems Science, 42(6), 951-958.

Wang,L. \& Dai, G.Z.(2009), Analysis of complex network: scale-free and its control (M), Beijin, Sciencep.

Wang, X. F., Li, X., \& Chen, G. R. (2006). Complex networks theory and its application [M]. Beijing: Qing Hua University Publication.

Wasko, M. M., Teigland, R. \& Faraj, S. (2009), The Provision of Online Public Goods: Examining Social Structure in an Electronic Network of Practice. Decision Support Systems. 47, pp. 254-265.

Watts, D. J. (2004). The" new" science of networks. Annual review of sociology, 243-270.

Watts, D. J., \& Strogatz, S. H. (1998). Collective dynamics of 'small-world'networks. Nature, 393(6684), 440-442.

Westland, J. C. (2010). Critical mass and willingness to pay for social networks. Electronic Commerce Research and Applications, 9(1), 6-19.

Wright, S. (1922). Coefficients of inbreeding and relationship. The American Naturalist, 56(645), 330-338. 